\title{
Methanol Fixation, but not Giemsa Staining, Inactivates Ebola and Lassa Viruses in Peripheral Blood Smears Made on Plastic Microscope Slides
}

\author{
Ryan F. Relich, ${ }^{1,2 \star}$ Heinz Feldmann, ${ }^{3}$ and Elaine Haddock ${ }^{3}$ \\ ${ }^{1}$ Department of Pathology and Laboratory Medicine, Indiana University School of Medicine, Indianapolis, Indiana; ${ }^{2}$ Division of Clinical \\ Microbiology, Department of Pathology and Laboratory Medicine, Indiana University Health, Indianapolis, Indiana; ${ }^{3}$ Division of Intramural \\ Research, Laboratory of Virology, National Institute of Allergy and Infectious Diseases, National Institutes of Health, Hamilton, Montana
}

\begin{abstract}
Diseases caused by many highly pathogenic viruses, including Ebola virus (EBOV) and Lassa virus (LASV), present with nonspecific signs and symptoms that overlap with common tropical diseases such as malaria. Initial diagnostic tests performed on patients under investigation for viral hemorrhagic fevers routinely include analysis of peripheral blood smears to detect and quantify Plasmodium species. In light of recent and ongoing Ebola virus disease and Lassa fever epidemics, clinical laboratories around the world require protocols for dealing with highly infectious specimens from patients with suspected or confirmed high-consequence diseases. Few validated protocols for safe analysis of peripheral blood smears are available, revealing a need for further research. In this study, we evaluated the performance of two plastic microscope slide types that offer safe alternatives to glass slides, determined the temporal parameters required to inactivate EBOV and LASV in thin blood smears by methanol fixation, and assessed the virucidal activity of Giemsa stain. Both types of plastic microscope slides performed optimally; there were no significant differences in blood cell morphology or tinctorial properties nor were differences noted in Plasmodium ovale morphology or staining, when compared with glass slides. For both EBOV and LASV, viable viruses were not detected in thin blood smears following fixation in absolute methanol for at least 2 minutes. By contrast, viable EBOV and LASV were recovered from all Giemsa-stained thick blood smears.
\end{abstract}

\section{INTRODUCTION}

The twenty-first century has witnessed the emergence and re-emergence of several highly pathogenic and highly infectious viruses. Currently, a novel Betacoronavirus, SARSCoV-2, is responsible for a pandemic that has caused nearly 18 million documented infections and approximately 700,000 deaths, globally. Previously encountered pathogens such as Ebola virus (EBOV) and Zika virus have recently caused epidemics of unprecedented proportions in geographic regions previously not known to be at risk for their spread. At the time of writing this article, an intensifying outbreak of Ebola virus disease (EVD) has surfaced in the northwestern corner of the Democratic Republic of the Congo (DRC) merely days after the world's second deadliest EVD epidemic, which was located in the northeastern part of the DRC, was declared over. Both the 2014-2016 West African and the 2018-2020 DRC EVD epidemics saw the movement of virus-infected individuals into neighboring countries; in the former, cases linked to individuals originating from countries experiencing local virus transmission reached regions outside of West Africa, including Europe and North America. ${ }^{1,2}$ As a consequence, secondary cases among healthcare personnel were documented in the United States. ${ }^{3}$

Lassa fever (LF), caused by Lassa virus (LASV), a rodentborne Mammarenavirus species, affects more than 500,000 individuals each year in West Africa, primarily in Sierra Leone, Guinea, Liberia, and Nigeria. Of those cases, approximately 5,000 patients succumb to hemorrhagic fever or other complications of LASV infection. ${ }^{4}$ Like EVD patients, LF patients are contagious throughout the duration of their illness and shed virions in blood and other body fluids. Because many LF

\footnotetext{
${ }^{*}$ Address correspondence to Ryan F. Relich, Division of Clinical Microbiology, Department of Pathology and Laboratory Medicine, Indiana University Health, 350 West 11th St., Rm. 6027E, Indianapolis,
} IN 46260. E-mail: rrelich@iupui.edu treatment centers lack consistent supplies and use of personal protective equipment that are required for barrier nursing, caregivers are at risk for contracting LASV while attending to patients. ${ }^{5}$ Like with EVD, exported cases of LF have also been reported, including a case in 2015 in New Jersey. ${ }^{6}$ The following year, the first case of LF transmission outside of Africa was documented in Germany in an undertaker who worked with the body of a deceased LF patient from Togo. ${ }^{7}$

Many viral hemorrhagic fevers, including EVD and LF, present with clinical signs and symptoms that overlap with those of common tropical diseases, especially malaria. To screen patients and determine the severity of parasitemia in cases of malaria, peripheral blood specimens must be microscopically examined by way of Giemsa-stained thick and thin smears. Microscopic examination is also required for morphological assessments and enumeration of different blood cell types, as it provides a measure of the patient's hematologic status at the time of specimen collection. Routine diagnostic laboratories are generally not equipped with the biocontainment infrastructure required to perform manipulations of specimens from patients known or suspected to have highconsequence infectious diseases, so many healthcare institutions have chosen to construct specialized biocontainment patient treatment units. Many of these facilities include on-site laboratories in which specimens can be handled. For the most part, biocontainment patient treatment unit laboratory support, either provided on-site or off-site, offers biosafety level-2 (BSL-2) and, rarely, BSL-3 containment. ${ }^{8}$ However, many of the protocols used by these laboratories, including peripheral blood smear fixation and examination, remain unstandardized, owing to a paucity of published data regarding safe work practices that can minimize threats posed by highly infectious clinical specimens in clinical laboratory settings.

Currently, the U.S. CDC and the American Society for Microbiology recommend methanol fixation of peripheral blood smears for 15 minutes before Giemsa staining 
(https://www.cdc.gov/vhf/ebola/laboratory-personnel/safespecimen-management.html), and the WHO recommends the addition of Triton- $X 100$ directly to blood specimens before smear preparation (http://www.wpro.who.int/mvp/ lab_quality/2096_oms_gmp_sop_07b.pdf). Other sources recommend 5-minute methanol fixation followed by 15 -minute $10 \%$ buffered formalin fixation or 30 -minute methanol fixation followed by heating at $95^{\circ} \mathrm{C}$ for 1 hour before staining. ${ }^{9}$ The disparity between these recommendations is largely due to a lack of published data regarding the parameters required to completely inactivate infectious EBOV and other highconsequence viral pathogens in peripheral blood smears. In addition, glass slides, which can readily break and puncture protective apparel and skin, are widely used in clinical laboratories, a practice that should be eliminated in these circumstances to minimize sharps-related injuries. To that end, two types of plastic microscope slides were evaluated to determine their suitability as alternatives to glass microscope slides for peripheral blood smear preparation. We also assessed the temporal parameters required to completely inactivate EBOV and LASV in peripheral blood smears by methanol fixation and evaluated the virucidal capacity of Giemsa stain using unfixed thick smears comprising spots of EBOV- or LASV-laden animal blood.

\section{MATERIALS AND METHODS}

Ethics and biosafety statement. All animal procedures were performed in strict accordance with the Guide for the Care and Use of Laboratory Animals (Office of Laboratory Animal Welfare, NIH, and the Animal Welfare Act). All manipulations of infectious substances, including experimentally infected animals, animal blood, and viral cultures, were performed according to Institutional Biosafety Committeeapproved protocols in the BSL-4 facility within the Integrated Research Facility (IRF), Rocky Mountain Laboratories, National Institute of Allergy and Infectious Diseases, $\mathrm{NIH}$, Hamilton, MT.

Evaluation of blood cell and Plasmodium appearance in Giemsa-stained plastic slides. To assess the suitability of polystyrene and acrylic microscope slides as surrogates for glass slides, remnant blood from a de-identified human patient infected with real-time PCR-confirmed Plasmodium ovale (parasitemia, 1.0\%) was used to prepare thin smears, as described in the following texts, on polystyrene and acrylic microscope slides (Caplugs Evergreen, Buffalo, NY). Thick smears were not prepared, as blood cell morphology assessments and definitive plasmodial identification are not possible using thick smears. The use of remnant de-identified patient specimens for such analyses was covered under an existing institutional review board-approved protocol (Indiana University Institutional Review Board study number IRB00000219). Air-dried smears were fixed for 2, 5, 10, 15, 30, and 60 minutes in absolute methanol to simulate possible fixation conditions that could be required to inactivate highconsequence viruses that could be present in blood smears. Fixed smears were stained with a working solution (1:50 dilution with tap water) of Giemsa stain (Sigma Aldrich, St. Louis, $\mathrm{MO}$ ) according to the manufacturer's specifications. Stained slides were examined by three microscopists trained to identify and quantitate Plasmodium spp. Observers were asked to comment on blood cell and plasmodial appearance (tinctorial properties and morphology) on plastic slides compared with those in smears prepared on glass slides. Representative images of blood cells and plasmodial forms were taken with an Olympus BX51 compound bright-field microscope equipped with an Olympus DP27 camera and cellSens imaging software (Olympus, Tokyo, Japan).

Blood specimens for inactivation studies. Ethylenediaminetetraacetic acid (EDTA)-anticoagulated whole blood was collected and mixed from two Zaire ebolavirus (EBOV) strain Mayinga (EBOV-Mayinga)-infected adult cynomolgus macaques that were used for separate EBOV studies by IRF personnel. Venipuncture was used to obtain samples at the time of routine health screenings. For LASV experiments, whole blood was collected and mixed from two Lassa mammarenavirus (LASV) strain Josiah (LASV-Josiah)infected cynomolgus macaques by venipuncture at the time of a routine health screening of animals used for a separate LASV study. Viral titers were determined by an endpoint dilution assay (for EBOV) or a focus-forming unit (FFU) assay (for LASV), which are described elsewhere. ${ }^{10,11} \mathrm{Un}$ adulterated nonhuman primate (NHP) blood specimens contained $2.9 \times 10^{5} 50 \%$ tissue culture infectious dose $\left(\right.$ TCID $\left._{50}\right) \mathrm{mL}^{-1}$ EBOV-Mayinga or approximately $5 \times 10^{3} \mathrm{FFUs}$ $\mathrm{mL}^{-1}$ LASV-Josiah. Initial experiments evaluating thin smears only were prepared using blood from an EBOVinfected NHP using 10- $\mu \mathrm{L}$ aliquots of well-mixed blood: smears contained $2.9 \times 10^{3} \mathrm{TCID}_{50}$ EBOV-Mayinga. Studies aimed to evaluate inactivation of LASV in both thin and thick smears were prepared using $10-$ and $40-\mu \mathrm{L}$ aliquots, respectively, of blood. Thin and thick smears contained 50 FFUs and 1,250 FFUs, respectively, LASV-Josiah.

To evaluate the ability of methanol and Giemsa stain to inactivate EBOV and LASV in blood smears containing higher titers of these viruses, blood specimens obtained from experimentally infected animals (for EBOV: EBOV-Mayingainfected CD-1 mice or an EBOV-Mayinga-infected NHP; for LASV: a LASV-Josiah-infected NHP) were spiked with culture-grown viruses. Following spiking of EBOV-Mayingainfected mouse blood with a concentrated $\left(1.5 \times 10^{8} \mathrm{TCID}_{50}\right.$ $\mathrm{mL}^{-1}$ ) stock of EBOV-Mayinga, thin smears were prepared using $10-\mu \mathrm{L}$ aliquots of spiked murine blood containing a total concentration of greater than $1.5 \times 10^{5} \mathrm{TCID}_{50}$ per slide for thin smears. Thick smears prepared using $40-\mu \mathrm{L}$ aliquots of EBOV-Mayinga-laden NHP blood contained greater than $6.0 \times 10^{6} \mathrm{TCID}_{50}$ per slide. For LASV spiking experiments, NHP blood was spiked with a stock $\left(5 \times 10^{5} \mathrm{FFUs} \mathrm{mL}^{-1}\right)$ of LASV-Josiah, and thin and thick smears made with 10- and $40-\mu \mathrm{L}$ of blood contained greater than $5.0 \times 10^{3}$ FFUs per slide and greater than $2.0 \times 10^{4}$ FFUs per slide, respectively.

Smear preparation, fixation, and/or staining. Thin smears were made by applying a $10-\mu \mathrm{L}$ drop of well-mixed infectious anticoagulated whole blood to one end of clean polystyrene and acrylic microscope slides (Caplugs). Smears were made according to a U.S. CDC-endorsed protocol. ${ }^{12}$ Thin smears were next air-dried and completely immersed in absolute (200 proof) methanol for 2, 5, 10, 15, 30, and 60 minutes; positive control smears were air-dried but were not methanol-fixed so as to not inactivate viruses. Negative controls, consisting of mock-infected Vero E6 cells suspended in phosphate-buffered saline (PBS), were air-dried but were not methanol-fixed. Because uninfected animal blood was not available at the time of experimentation, Vero E6 cells were used as a substitute. Fixation at all time points was repeated 
in triplicate for both polystyrene and acrylic slide types. Following fixation for the prescribed time period, smears were airdried within a biological safety cabinet.

Thick smears were prepared by applying $40-\mu \mathrm{L}$ aliquots of infectious EDTA-anticoagulated blood to both polystyrene and acrylic microscope slides and smearing the blood in a circular motion with the pipet tip until they were $1-2 \mathrm{~cm}$ in diameter, according to the CDC protocol. ${ }^{12}$ Smears were next air-dried and then overlaid with $0.5 \mathrm{~mL}$ of a working Giemsa stain solution. Smears were stained for 10 minutes and 40 minutes; each treatment was performed twice more to generate triplicate data. Unstained thick smears were prepared using infectious blood or mock-infected Vero E6 cells suspended in PBS to generate positive and negative controls, respectively, as described earlier.

Viral infectivity assay. To assess the viability of EBOVMayinga and LASV-Josiah following methanol fixation or Giemsa staining, fixed or Giemsa-stained smears were removed from slides with flocked swabs, suspended in PBS, and overlaid onto monolayers of Vero E6 cells. In brief, for methanol-fixed thin smears, sterile PBS-moistened flocked swabs were used to entirely remove smears from microscope slides. Blood was eluted from swabs into 1.5-mL aliquots of PBS by vortexing swab tips in sealed tubes. Resulting eluates were evenly distributed over three wells of Vero E6 cell monolayers in six-well cell culture plates and permitted to adsorb for 1 hour at $37^{\circ} \mathrm{C}$. Following adsorption, $2.5-\mathrm{mL}$ aliquots of Dulbecco's Modified Eagle Medium containing L-glutamine, $2 \%$ fetal bovine serum, penicillin, and streptomycin (infection medium) was added to each well. Cells were next incubated for at least 14 days at $37^{\circ} \mathrm{C}$ in $5 \% \mathrm{CO}_{2}$, and cells that lacked demonstrable cytopathic effects (CPEs) were passaged by transferring $1-\mathrm{mL}$ aliquots of culture medium to plates of fresh cells and fresh infection medium. This process was completed one additional time before CPE-less wells from the original cultures were deemed negative.

To assess the viability of viruses in Giemsa-stained experimental treatments, the entire volume $(0.5 \mathrm{~mL})$ of Giemsa stain used to stain slides and the thick smears was harvested and used to inoculate Vero E6 cells. Cell culture plates were incubated, passaged, and analyzed as before. GraphPad Prism version 8.1.0 (GraphPad; available at: https://www.graphpad. com/scientific-software/prism/) was used for constructing graphs of viral viability for each treatment.

\section{RESULTS}

Polystyrene and acrylic microscope slides are suitable and safe alternatives to glass microscope slides. Glass microscope slides present physical hazards (e.g., cuts and punctures) that increase the risk of high-consequence pathogen transmission in the laboratory environment, so, when possible, safer plastic alternatives should be used. To determine if plastic slides could serve as replacements for glass slides for peripheral blood smear examination and plasmodial identification, we prepared thin peripheral blood smears using EDTA-anticoagulated whole blood from a human patient infected with $P$. ovale. Slides fixed for various lengths of time (2-60 minutes) were stained with Giemsa. Observers, comprised three doctoral-level clinical microbiologists who were blinded to fixation times and slide types, were asked to compare erythrocyte, leukocyte, and $P$. ovale-infected cell morphologies and staining characteristics in smears prepared on the different slide types. In each case, observers could not identify significant differences in blood cell and plasmodial morphologies or staining qualities on smears prepared on plastic slides that were fixed for various times. However, one observer noted a very slight increase in the number of dacrocytes ("teardrop" erythrocytes) on a few acrylic slides. A correlation between experimental treatments and the perceived appearance of more dacrocytes could not be established. These observations are in line with those of a previous study that showed prolonged methanol fixation did not adversely affect the interpretability of peripheral blood smears stained with Wright's stain. ${ }^{13}$ Photographs of representative fixation time points and slide materials are shown in Figure 1.

Methanol fixation rapidly inactivates infectious EBOV and LASV in thin peripheral blood smears. Peripheral blood samples containing infectious EBOV-Mayinga and LASVJosiah were used in separate experiments to determine the temporal parameters required for inactivation of these viruses by absolute methanol, the most commonly used fixative for the preparation of thin smears destined for parasitological and hematological analyses. Blood smears containing infectious EBOV-Mayinga at titers of $2.9 \times 10^{3}$ and greater than $1.5 \times 10^{5}$ $\mathrm{TCID}_{50}$ per smear or LASV-Josiah at titers of 50 and greater than $5 \times 10^{3}$ FFUs per smear were prepared as described using either NHP or murine blood. Air-dried smears were fixed for various amounts of time and air-dried before smear removal and culturing. For all of the fixation time points assessed (2-60 minutes) and for all the blood specimen types (natural infections and spiked, higher titer samples) analyzed, viable EBOV-Mayinga and LASV-Josiah were not detected (Figure 2). Positive and negative controls were performed as expected. In positive control slides, EBOV-Mayinga and LASV-Josiah CPEs were readily apparent within the first 14 days of incubation, and cells receiving mock-infected Vero E6 cells remained negative following two additional passages, as with the experimental treatments (Figure 2).

Giemsa staining alone does not inactivate EBOV and LASV in thick peripheral blood smears. Thick smears made with $40-\mu \mathrm{L}$ aliquots of animal blood containing EBOVMayinga at a titer of greater than $6.0 \times 10^{6} \mathrm{TCID}_{50}$ per smear or LASV-Josiah at titers of greater than $2.0 \times 10^{4} \mathrm{FFUs} \mathrm{per}$ smear were prepared as previously described. After drying within the biosafety cabinet, smears were covered with a working solution of Giemsa stain for 10- and 40-minute exposure times. Following incubation, the Giemsa stain and peripheral blood spots were harvested from slides and were used as inocula for monolayers of Vero E6 cells in six-well plates. Thick smears containing either EBOV-Mayinga or LASV-Josiah were positive for viral growth within 14 days of inoculation (Figure 2). Positive and negative controls similar to those generated for methanol fixation experiments were used in this series of experiments and yielded expected results, as before (Figure 2). Data generated by these experiments demonstrated that Giemsa staining alone is insufficient for inactivation of EBOV and LASV in thick smears, so the analysis of thick smears should be avoided to minimize the chances of laboratory-acquired infections. Although exclusion of thick smears is essential for avoiding hazards posed by highconsequence pathogens, readers should be aware that the overall sensitivity of the microscopic detection of Plasmodium $\mathrm{sp}$. will be reduced, especially in cases of low-level 


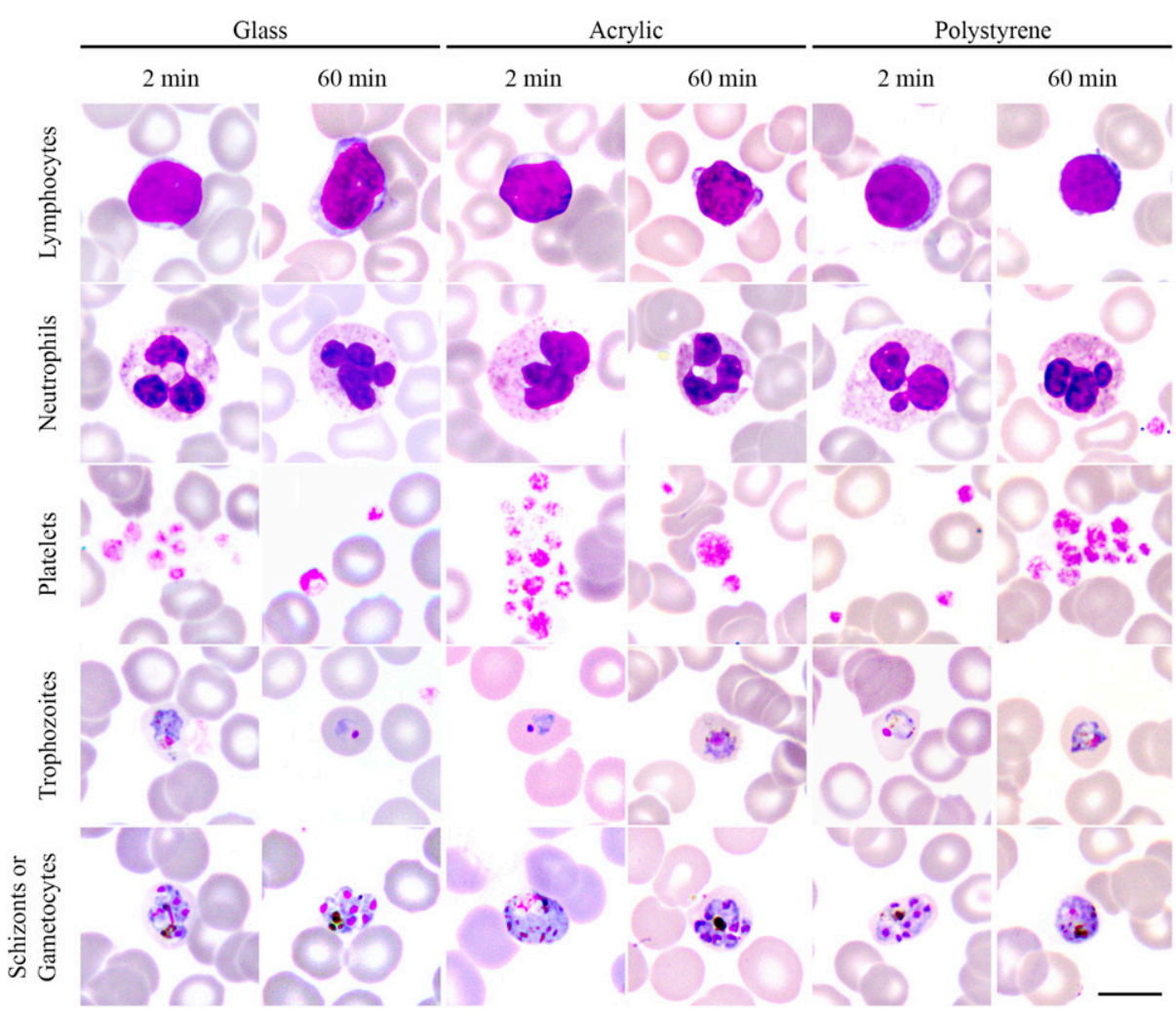

FIGURE 1. Morphological and tinctorial properties of blood cells and plasmodial forms in Giemsa-stained thin blood smears prepared on different slide types and methanol-fixed smears for varying lengths of time. No significant differences in blood cell or plasmodial morphologies, and only minor differences in staining were observed. Despite infection of the specimen source-patient with Plasmodium ovale, Schüffner's stippling was not evident; however, stippling was also not observed on slides used for the initial diagnosis of the patient's malaria. Bar, $10 \mu \mathrm{m}$.

parasitemia. In an effort to maximize the likelihood of detecting plasmodia under these circumstances, laboratorians should consider examining additional thin smears. Alternately, laboratorians may wish to explore PCR-based testing options, which will obviate the need for smear preparation and its associated hazards.

\section{DISCUSSION}

The emergence and re-emergence of numerous highly pathogenic viruses such as EBOV and LASV have prompted healthcare facilities around the globe to establish protocols for safe handling of patients, blood and other body fluids, and infectious wastes generated as part of the diagnosis and treatment of suspected or confirmed infections. The broad use of glass microscope slides, which present physical hazards that could potentially expose laboratorians to significant biohazards, coupled with a lack of standardized methods that have been proven to totally inactivate EBOV and LASV in peripheral blood smears, leaves holes in the safety infrastructure of laboratories charged with the tasks of performing malaria testing on patients under investigation for EVD and LF. The use of lateral-flow immunochromatographic malaria diagnostic assays in place of blood smears is an option; however, these methods are known to have drawbacks, including low analytical sensitivities and misclassification of some Plasmodium spp. ${ }^{14}$ In addition, parasite burden cannot be obtained by lateral-flow tests, so peripheral blood smears must be examined to make this estimation. The use of nucleic acid amplification tests such as PCR presents another option; however, most clinical laboratories do not routinely perform this testing, so specimens would have to be referred to another laboratory for testing.

To address these issues, we investigated the suitability of two plastic microscope slide types, polystyrene and acrylic, as alternates to glass microscope slides; evaluated temporal parameters required to totally inactivate EBOV and LASV in thin blood smears by methanol fixation; and assessed the virucidal activity of Giemsa stain. We showed that regardless of the fixation times tested, Giemsa-stained thin smears using both plastic slide types produced results equivalent to those obtained using glass slides. We therefore suggest the substitution of glass slides with either polystyrene or acrylic slides for use in laboratories that perform peripheral blood smear examinations on patients under investigation for EVD and LF and potentially other viral hemorrhagic fevers. For each methanol fixation time point assessed, complete inactivation of EBOV and LASV was observed in all experimental replicates, and Giemsa staining alone was determined to be insufficient to render thick blood smears safe to handle outside of maximum biocontainment.

Previous studies conducted by Cutts et al. ${ }^{15}$ at the Canadian Science Centre for Human and Animal Health laid the groundwork for a better understanding of the dynamics of EBOV inactivation in peripheral blood thin smears. Their work determined that absolute methanol completely inactivated EBOV-Makona after 15 minutes of exposure. Our investigations add to that work by providing more precise inactivation 


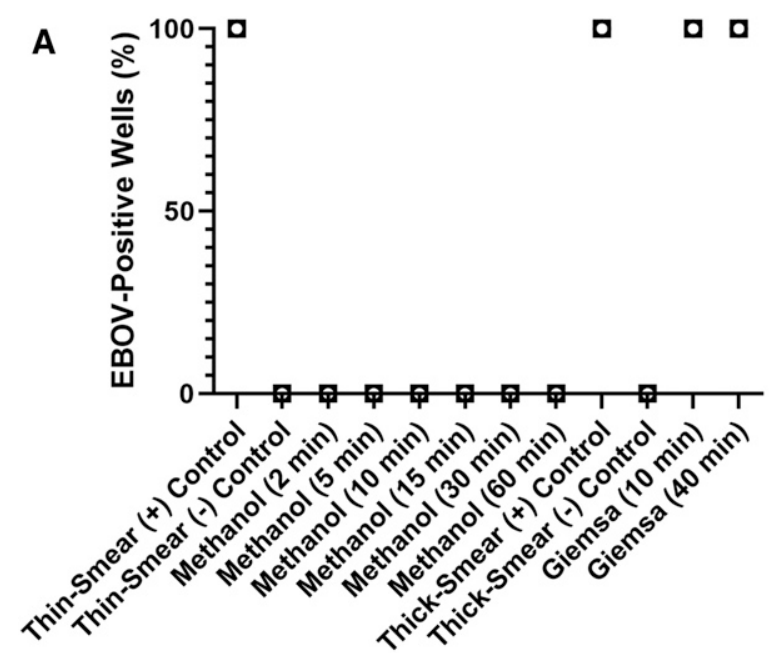

\section{- EBOV-Infected NHP Blood \\ ㅁ EBOV-Spiked Blood}

\section{Experimental Conditions}



\section{- LASV-Infected NHP Blood}

口 LASV-Spiked Blood

\section{Experimental Conditions}

FIGURE 2. (A) Infectious Ebola virus (EBOV) and (B) Lassa virus (LASV) were undetectable in thin blood smears fixed in absolute methanol for each time point tested in all replicates. Infectious viruses were detected in all Giemsa-stained thick blood smears for both time points tested in all replicates.

times and include another high-consequence virus, LASV. In addition, we have shown that Giemsa stain is not an effective virucide. This knowledge, coupled with the fact that thick smears require a larger volume of blood, indicates that thick smears should not be performed because they constitute an infection hazard following staining. Because thin smear-only examination reduces the analytical sensitivity of smear examination-based malaria diagnosis, additional thin smears should be examined or an alternative method (e.g., PCR) should be used to screen blood specimens.

Limitations of this study include the use of a single blood specimen from one patient with low-level $P$. ovale parasitemia and a lack of evaluation of blood cell and plasmodial appearance in Giemsa-stained smears prepared on plastic slides using blood specimens from patients with different levels of parasitemia. Because the prevalence of malaria among patients treated at the hospital from which the human blood specimen was obtained for this study is very low, only the single $P$. ovale-containing specimen was available during this study. Despite this shortcoming, this study describes detailed methods that clinical laboratories that choose to evaluate these smear fixation and morphological analyses can replicate using blood specimens from patients infected with other Plasmodium spp. (e.g., Plasmodium falciparum). In addition, it may be possible for other investigators to evaluate variable fixation times on the ability to calculate parasitemia.

In the future, we plan to investigate additional laboratory practices that can mitigate the hazards associated with manipulations of other clinical specimens containing highconsequence viruses. For example, we are interested to know if routine Gram staining of positive blood culture broths, respiratory secretions, and other body fluids is sufficient to 
inactivate EBOV, LASV, Crimean-Congo hemorrhagic fever virus, Nipah virus, and others. Until then, we offer the data from this set of experiments, which enrich the knowledge base of safety practices used for the diagnosis of malaria and other blood parasite infections in individuals under investigation, or care, for EVD and LF. Such data can be used in conjunction with thorough risk assessments for the development of protocols to be used in biocontainment patient care laboratories or other clinical laboratories charged with the tasks of assisting with the care of highly infectious patients.

Received November 8, 2019. Accepted for publication August 13, 2020.

Published online September 21, 2020.

Acknowledgments: We wish to thank researchers at Rocky Mountain Laboratories for donation of NHP and mouse blood used in these experiments and medical laboratory personnel at the Sidney and Lois Eskenazi Hospital in Indianapolis, IN, for provision of $P$. ovalecontaining blood. In addition, we thank B. Schmitt, K. Nagaro, and T. Phan (IU School of Medicine, Indianapolis, IN) for reviewing Giemsastained blood thin smears on plastic slides, and B. Pritt and the Mayo Clinic Diagnostic Parasitology Laboratory (Mayo Clinic, Rochester, MN) for definitive identification of $P$. ovale by real-time PCR in the blood specimen used for experimental analysis.

Financial support: This work was supported by the Division of Intramural Research, National Institute of Allergy and Infectious Diseases, $\mathrm{NIH}$.

Authors' addresses: Ryan F. Relich, Department of Pathology and Laboratory Medicine, Indiana University School of Medicine, Indianapolis, IN, and Division of Clinical Microbiology, Department of Pathology and Laboratory Medicine, Indiana University Health, Indianapolis, IN, E-mail: rrelich@iupui.edu. Heinz Feldmann and Elaine Haddock, Division of Intramural Research, Laboratory of Virology, National Institute of Allergy and Infectious Diseases, National Institutes of Health, Hamilton, MT, E-mails: heinrich.feldmann@nih.gov and elaine.haddock@nih.gov.

\section{REFERENCES}

1. Lopaz MA et al.; Working Group of Ebola Investigation Team of Madrid, 2015. First secondary case of Ebola outside Africa: epidemiological characteristics and contact monitoring, Spain, September to November 2014. Euro Surveill 8: 21003.

2. Chevalier MS et al., 2014. Ebola virus disease cluster in the United States - Dallas County, Texas, 2014. MMWR Morb Mortal Wkly Rep 63: 1087-1088.
3. Eddy C, Sase E, 2015. The 2014 Dallas, Texas, Ebola incident: global implications to all-hazards preparedness and health care worker protection. J Environ Health 78: 28-32.

4. Bausch DG, Mills JN, 2014. Arenaviruses: Lassa fever, Lujo hemorrhagic fever, lymphocytic choriomeningitis, and the South American hemorrhagic fevers. Kaslow RA, Stanberry LR, Le Duc JW, eds. Viral Infections of Humans. Boston, MA: Springer.

5. Grahn A, Bråve A, Tolfvenstam T, Studahl M, 2018. Absence of nosocomial transmission of imported Lassa fever during use of standard barrier nursing methods. Emerg Infect Dis 24: 978-987.

6. Kulkarni PA et al., 2018. Case report: imported case of Lassa fever - New Jersey, May 2015. Am J Trop Med Hyg 99: 1062-1065.

7. Ehlkes L, George M, Samosny G, Burckhardt F, Vogt M, Bent S, Jahn K, Zanger P, 2017. Management of a Lassa fever outbreak, Rhineland-Palatinate, Germany, 2016. Euro Surveill 22: 16-00728.

8. Garibaldi BT, Chertow DS, 2017. High-containment pathogen preparation in the intensive care unit. Infect Dis Clin North Am 31: 561-576.

9. Public Health Laboratory Network, 2014. Laboratory Procedures and Precautions for Samples Collected from Patients with Suspected Viral Haemorrhagic Fevers. Canberra, Australia: Australian Government Department of Health. Available at: https://www1.health.gov.au/internet/main/publishing.nsf/ Content/cda-pubs-other-vhf.htm. Accessed September 9, 2019.

10. Marzi A, Feldmann F, Hanley PW, Scott DP, Günther S, Feldmann $\mathrm{H}, 2015$. Delayed disease progression in Cynomolgus macaques infected with Ebola virus Makona strain. Emerg Infect Dis 21: 1777-1783.

11. Weidmann M, Sall AA, Manuguerra J-C, Koivogui L, Adjami A, Traoré FF, Hedlund K-O, Lindegren G, Mirazimi A, 2011. Quantitative analysis of particles, genomes and infectious particles in supernatants of haemorrhagic fever virus cell cultures. Virol J 8: 81.

12. CDC, 2019. Laboratory Diagnosis of Malaria: Preparation of Blood Smears. Atlanta, GA: CDC. Available at: https://www.cdc.gov/ $\mathrm{dpdx} /$ resources/pdf/benchAids/malaria/Malaria_procedures_ benchaid.pdf. Accessed March 20, 2020

13. Lozier JN, Calvo KR, 2015. Effect of viral decontamination measures on wright-stained blood smears. Blood 125: 1350-1351.

14. DiMaio MA, Pereira IT, George TI, Banaei N, 2012. Performance of the BinaxNOW for diagnosis of malaria in a U.S. hospital. J Clin Microbiol 50: 2877-2880.

15. Cutts T, Cook B, Poliquin G, Strong J, Theriault S, 2016. Inactivating Zaire ebolavirus in whole-blood thin smears used for malaria diagnosis. J Clin Microbiol 54: 1157-1159. 